\title{
Sinoatrial Response to Premature Atrial Stimulation during Atrial Pacing in Aged Patients with and without Sinus Node Dysfunction
}

\author{
Keiji Ueda, M.D., * Kan Takayanagr, M.D.,** \\ Hiroshi Matsuo, M.D., ${ }^{* *}$ Takao Okımoto, M.D., ${ }^{* * *}$ \\ Junichiro Mifune, M.D., * Shinichiro Ohkawa, M.D.,* \\ Chizuko Kamata, M.D., * and Masaya Sugrura, M.D.*
}

\section{SUMmary}

Sinus node function was analysed in 15 aged patients, including 8 with sick sinus syndrome and 7 without apparent sinus node dysfunction, by rapid atrial pacing and premature atrial stimulation during sinus rhythm and 5 beats of atrial pacing.

Three patterns of sinoatrial response were identified by premature atrial stimulation during atrial pacing. Type I response (3 cases) represented approximately constant return cycles and type II (2 cases) represented a markedly prolonged return cycles following premature stimulation. Type III response was characterized by a progressive shortening of return cycles as the prematurity of atrial extrastimuli was increased.

Underlying mechanisms responsible for these responses were discussed in relation to the sinus node automaticity and sinoatrial conduction.

\section{Additional Indexing Words:}

Sinus node recovery time Sinoatrial conduction Incomplete interpolation

$I^{\prime}$

n recent years growing interest has been focussed on electrophysiologic evaluation of sinus node dysfunction in man. ${ }^{1-8)}$ The basic disorders responsible for the syndrome of sinus node dysfunction (sick sinus syndrome) are sinus arrest and/or sinoatrial exit block, and a variety of interventions have been offered as a means of identifying sinus node dysfunction. The assessment of these interventions in man, especially in those who do not have fullblown features of the syndrome, requires further prospective study in a large

From the Division of Cardiology,* Tokyo Metropolitan Geriatric Hospital, the Second Department of Internal Medicine** and the Department of Physical Therapy and Medicine,*** Faculty of Medicine, University of Tokyo, Tokyo.

Address for reprint request: Keiji Ueda, M.D., Division of Cardiology, Tokyo Metropolitan Geriatric Hospital, Itabashi-ku, Tokyo 173, Japan.

Received for publication January 18, 1978. 
number of patients with suspected sick sinus syndrome. ${ }^{8)}$

Dhingra and coworkers ${ }^{9}$ reported the sinus node responses to atrial extrastimuli during atrial pacing in patients without apparent sinus node disease and they demonstrated marked facilitation of sinus nodal reentry and interpolation in these cases. It was the purpose of this study to report the sinoatrial responses to atrial extrastimuli during atrial pacing in aged patients including those with sinus node dysfunction with an attempt to evaluate the usefulness of the technique in the understanding of underlying mechanism in this syndrome.

\section{Materials and Methods}

Materials: The study group consisted of 15 patients who were studied during diagnostic electrophysiologic catheterization. The mean age of the patients was 76.7 years (range 66 to 86 ); there were 7 men and 8 women. The patients were divided into following 2 groups: Group A; 8 cases with sick sinus syndrome (SSS), including 4 with marked sinus bradyarrhythmia, 2 with recorded episodes of transient sinus arrest, and 2 with bradycardia-tachycardia syndrome and Group B; 7 cases without apparent sinus node dysfunction, including 2 cases with atrioventricular block, and 5 with right or left bundle branch block. The clinical data and electrocardiographic diagnosis are shown in Table $\mathrm{I}$.

Methods: The patients were studied in the resting, nonsedated, and postabsorptive state. No patient was taking cardiac drugs at the time of the study.

Two electrode catheters were inserted into the right atrium (RA). One catheter, quadripolar or bipolar, was placed at the junction of the superior vena cava and the right atrium and distal 2 electrodes of a quadripolar electrode catheter were used for stimulation of RA, while proximal 2 electrodes for the recording electrograms of high RA. When a bipolar electrode catheter was used, electrogram of high RA was not recorded. The second (bipolar) catheter was placed near the tricuspid valve to record low atrial electrogram and His bundle electrogram.

Electrocardiographic leads $\mathrm{I}, \mathrm{aV}_{\mathrm{F}}$, and $\mathrm{V}_{1}$ were simultaneously recorded. Recordings were obtained on a multichannel oscilloscopic recorder (Electronics for Medicine, DR-12) at paper speed of 50 and $100 \mathrm{~mm} / \mathrm{sec}$ and also on a magnetic tape recorder.

Two types of atrial stimulation were performed: the atrial pacing with twice the diastolic threshold was performed by a cardiac pacemaker for measurement of maximum sinus node recovery time expressed in percent $\left(\mathrm{SRT}_{\max }\right.$ ) as previously described. ${ }^{6}$ Subsequently atrial pacing (AP) was performed for 5 beats with intervals slightly shorter than the sinus cycle length. The premature atrial stimulation (PAS), twice the diastolic threshold and $3 \mathrm{msec}$ in duration, was performed by a programmable stimulator (Nihon Koden MDS-2HS) after every fifth sinus or paced beat with progressively decreasing coupling intervals by 10 to $20 \mathrm{msec}$ until atrial capture was lost.

During sinus rhythm, the whole range of sinus cycle length was scanned by PAS. During AP, scanning was begun at prematurity equivalent to approximately $90 \%$ of pacing cycle in 11 cases and in 4 cases at the coupling interval which was 
slightly shorter than pre-paced sinus cycle length. When AP was applied, it was discontinued after 5 paced atrial beats for measurement of "escape intervals" and after 5 paced beats followed by PAS with varying coupling intervals for measurement of return cycles. Three different pacing rates were used in 2 cases, 2 different rates in 6 , and a single rate in 7.

Following atrial intervals were measured; (1) spontaneous sinus cycle length (SCL), i.e., the interval between the last 2 spontaneous sinus $\mathrm{P}$ waves that preceded PAS; (2) the coupling interval (CI), i.e., the interval between the last $P$ wave (sponteneous or paced) and PAS; (3) the return cycle length (RC), i.e., the interval between PAS and subsequent sinus P wave; and (4) escape interval i.e., the interval between the last paced $P$ wave and subsequent sinus $P$ wave when AP was discontinued after 5 paced atrial beats without PAS. CI, RG, and escape interval were divided by SCL for comparison and normalized $\mathrm{RC}$ or escape interval was plotted (ordinate) against normalized CI (abscissa) in each case.

On the plot of RC against CI for PAS during sinus rhythm, compensatory zone (zone of interference) and reset zone were identified as defined by Dhingra et al. ${ }^{9)}$ When compensatory zone was followed by reset zone, as the prematurity of PAS was increased, the response was designated as type A response as previously reported. ${ }^{7}$ When $\mathrm{RC}$ followed the compensatory line without showing plateau of reset zone, it was designated as type $B_{1}$ response and when $\mathrm{RC}$ following the compensatory zone was shorter than the compensatory line, but longer than reset line, it was designated as type $B_{2}$ response.")

Sinoatrial conduction time (SACT), calculated by the method of Strauss et al.,2) was obtained by measuring the difference between $\mathrm{RC}$ in reset zone and SCL.

Atrial functional refractory period was defined as reported by Denes et al. ${ }^{10}$

\section{Results}

1) The maximum sinus node recovery time $\left(S R T_{\max }\right.$ ) (Table $\mathrm{I}$ )

$\mathrm{SRT}_{\max }$ in 8 patients of group A (SSS) ranged from 146 to $635 \%$ with an average of $231 \%$ and in 5 cases it exceeded $160 \%$. In 7 cases of group B, $\mathrm{SRT}_{\max }$ ranged from 120 to $154 \%$ with an average of $135 \%$.

2) Sinoatrial responses to PAS during sinus rhythm (Table I)

In 4 cases of Group A and in 6 cases of Group B, PAS delivered in late diastole was followed by fully compensatory return cycles and progressive shortening of CI resulted in nearly constant duration of RG (type A response). In 3 other cases of Group A, the plot of normalized RC against normalized CI did not show the plateau after the transition from the compensatory line, but showed progressive lengthening with increasing prematurity of PAS. The plot of $\mathrm{RC}$ in these cases fell either on the compensatory line (type $\mathrm{B}_{1}$ response) or between the compensatory line and reset line (type $\mathrm{B}_{2}$ response). In the remaining 2 cases of Group $A$, distribution of $R C$ showed scattering due to the presence of marked sinus arrhythmia and did not show any relation to the prematurity of PAS. 


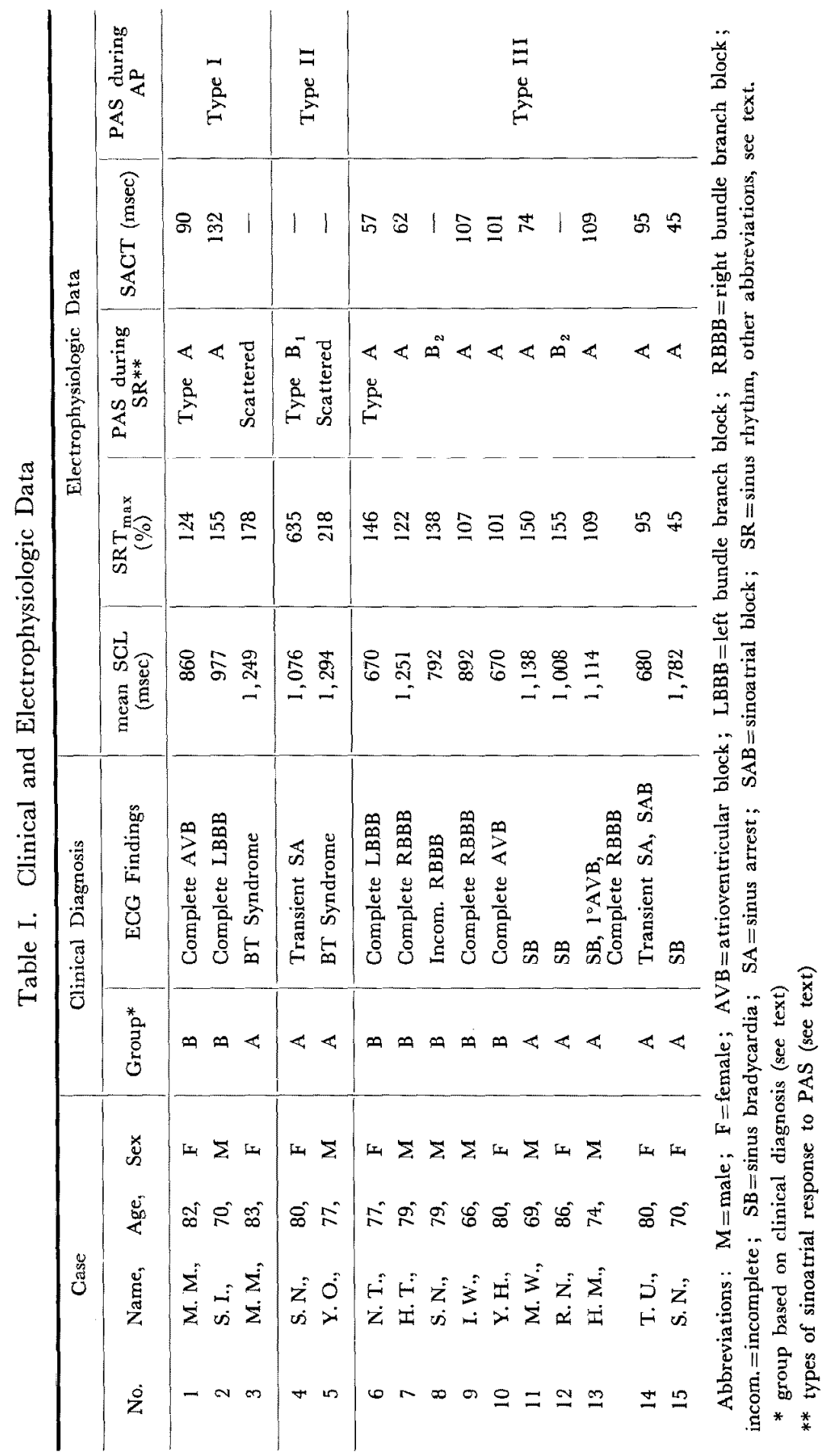


3) Sinoatrial conduction time (SACT) (Table I)

SACT could be calculated in 10 cases. The average SACT in 4 cases of Group A was $80.8 \mathrm{msec}$ (range 45 to $109 \mathrm{msec}$ ), while in 6 cases of Group B it was $91.5 \mathrm{msec}$ (range 57 to $132 \mathrm{msec}$ ).

4) Sinoatrial responses to PAS during AP (Table I)

Following 3 different patterns were observed.

Type I response (Fig. I): In 3 cases (cases No. 1-3), RG remained relatively constant except those following very early PAS. Compensatory zone was not observed when cycle length of AP was slightly shorter than SCL.

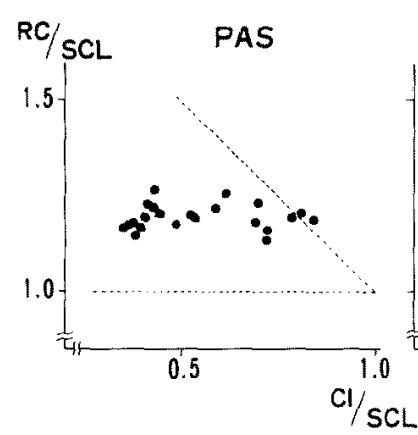

(a)

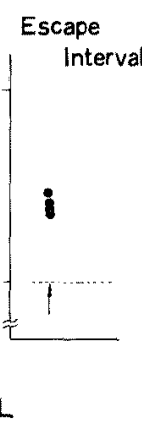

(b)

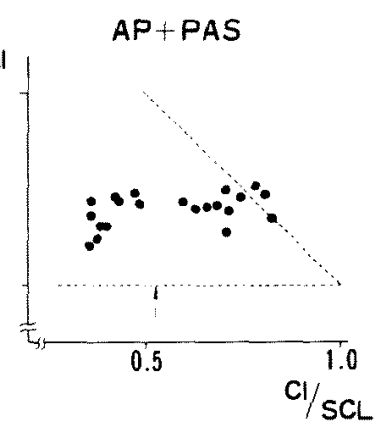

(c)

Fig. 1. Type I response in Case No. 1.

Normalized return cycle ( $R C$ ) is plotted against normalized coupling interval (CI). (a) PAS during sinus rhythm, (b) escape interval, and (c) PAS during atrial pacing (pacing cycle length, $461 \mathrm{msec}$ ). Arrows indicate pacing cycle length.
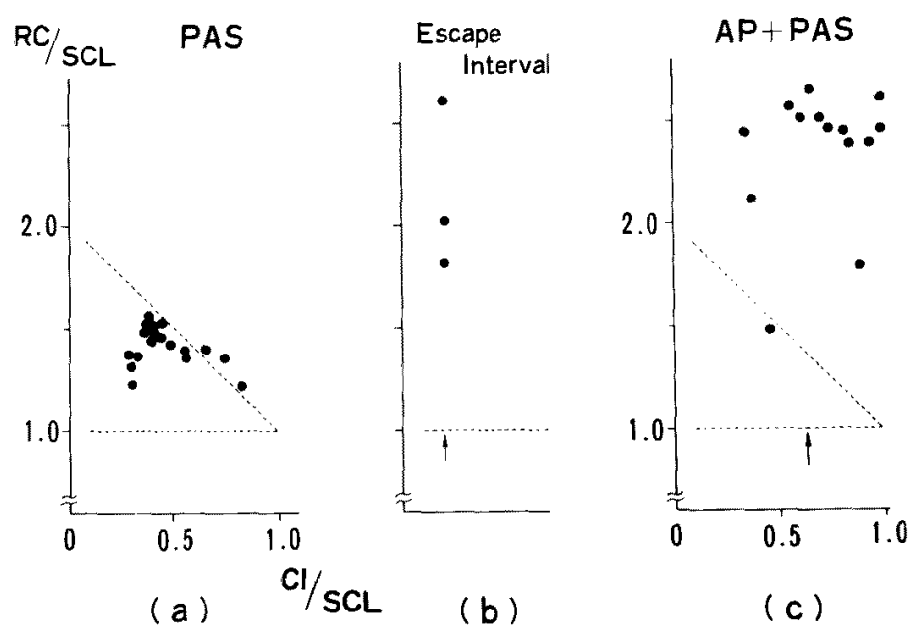

Fig. 2. Type II response in Case No. 4.

(a) PAS during sinus rhythm, (b) escape interval, and (c) PAS during atrial pacing (pacing cycle length, $667 \mathrm{msec}$ ). Abbreviations, see legend of Fig. 1. 
In cases No. 1 and 2, RCs during AP were approximately identical to those observed during sinus rhythm. In the remaining case (case No. 3), no reset zone could be defined during sinus rhythm because of variability in SCL and inconsistency of normalized $\mathrm{RC}$. However, $\mathrm{RG}$ during $\mathrm{AP}$ remained relatively constant and reset zone could be defined.

Type II response: In 2 cases (cases No. 4 and 5), RC during AP showed a marked prolongation regardless of the prematurity of PAS compared to that during sinus rhythm, as were escape intervals (Fig. 2).

Type III response: In 10 cases (cases No. 6 to 15) compensatory zone as identified during sinus rhythm was not observed and RC during AP showed gradual shortening with progressive increase in the prematurity of PAS, and ultimately instances of RC equal or nearly equal to SCL could be encountered as shown in Fig. 3.

Sinoatrial response of type III showed characteristic features; (1) RG after PAS during AP in late diastole was prolonged compared to that during sinus rhythm, whereas RG after PAS in earlier diastole showed progressive shortening in approximately linear fashion as the prematurity of PAS was increased; (2) progressively shortened RG in earlier diastole remained longer than SCL, except that showing precipitous and marked shortening in echo zone; and (3) the zone showing gradual shortening of $\mathrm{RG}$ accounted for $26.7 \%$ (range: $17-48 \%$ ) of SCL on an average. The outer limit of the
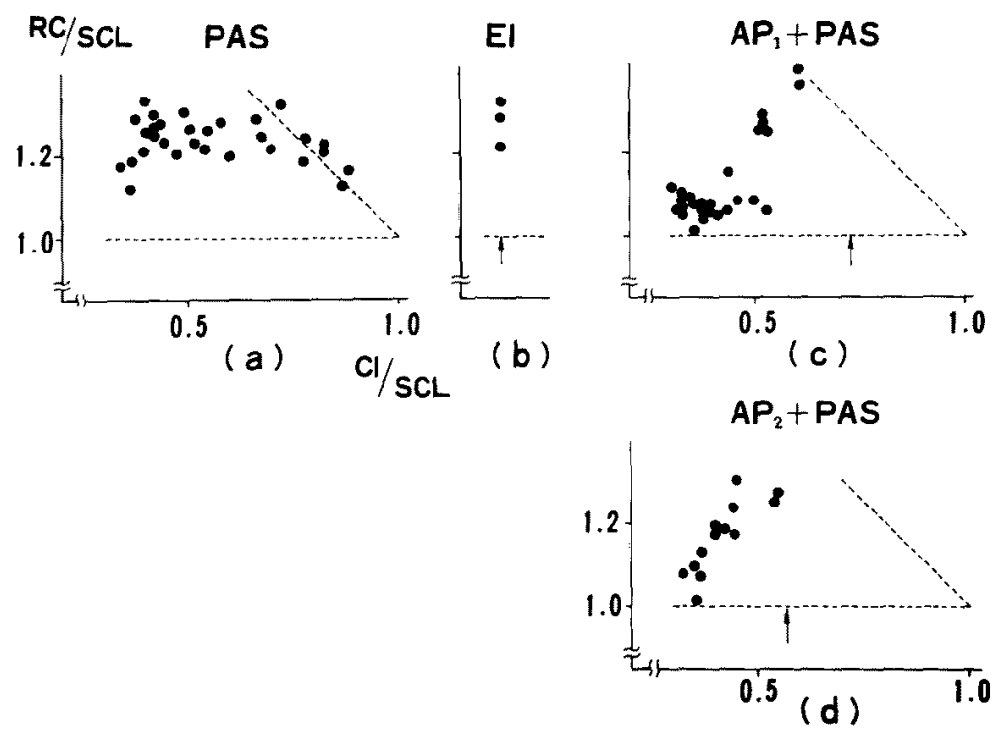

Fig. 3. Type III response in Case No. 9.

(a) PAS during sinus rhythm, (b) escape interval, (c) PAS during atrial pacing (pacing cycle length, $667 \mathrm{msec}$ ), and (d) PAS during atrial pacing (pacing cycle length, $500 \mathrm{msec}$ ). Abbreviations, see legend of Fig. 1. 


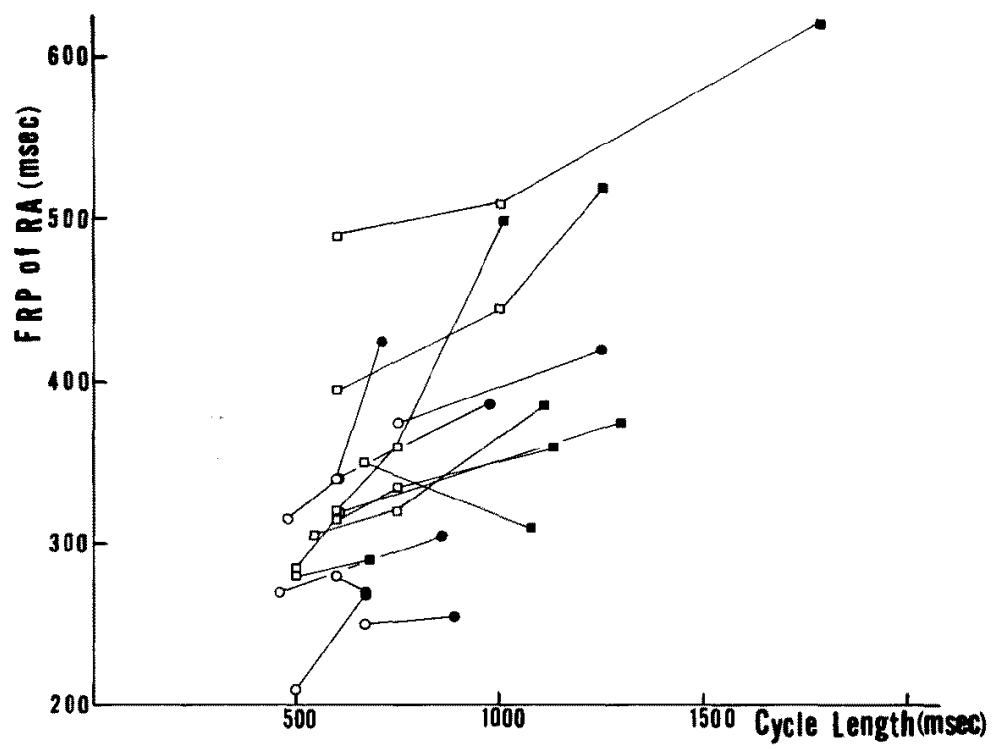

Fig. 4. Relation between functional refractory period (FRP) of the right atrium (RA) and sinus (closed squares and circles) or pacing (open squares and circles) cycle length. Squares indicate cases of Group A (SSS) and circles indicate those of Group B.

zone was $61.3 \%$ on an average (range: $49-85 \%$ ) of SCL and the inner limit of the zone was $34.6 \%$ (range: $26-49 \%$ ) of SCL.

The numbers of cases showing each of these 3 patterns of sinoatrial response to PAS in Groups A and B were as follows; type I response in 1 case in Group A and 2 in Group B, type II response only in 2 in Group A and type III response in 5 cases in Group A and 5 in Group B.

5) The functional refractory period of RA (FRP $P_{R A}$ ) (Fig. 4)

FRP $_{\mathrm{RA}}$ during AP was shorter than that during sinus rhythm in 13 cases. In the remaining 2 cases, FRP $_{R A}$ showed a slight prolongation during $\mathrm{AP}$ compared to that during sinus rhythm. In 8 cases in whom AP was repeated with progressively shorter pacing intervals, $F P_{R_{A}}$ showed further shortening.

\section{Discussion}

It has been previously demonstrated in both animals and man that PAS exerts variable effect on the atrial rhythm. ${ }^{2), 7), 11), 12)}$ Ticzon et al $^{(2)}$ showed in dogs that sinus nodal responses to PAS could be categorized into 4 zones defined by $\mathrm{RC}$ in relation to the prematurity of PAS. Dhingra et al ${ }^{9}$ reported that 4 zones as shown in animals could be defined in patients without 
apparent sinus node dysfunctiou. Progressive lengthening of RC with increasing prematurity of PAS, not showing relatively constant $R C$ in earlier diastole, has also been demonstrated in patients with $\mathrm{SSS}^{2)}$ and in aged cases without clinically apparent sinus nodal disease. ${ }^{\text {7) }}$

In the present study of aged cases, 3 different patterns of sinoatrial responses to PAS, designated as types I, II, and III, were observed during AP. Type $I$ response, in which $\mathrm{RC}$ remained relatively constant as in reset zone during sinus rhythm, suggested that (1) sinoatrial node automaticity did not change during 5 beats of AP with intervals slightly shorter than SCL and (2) antegrade and retrograde conduction time remained constant during AP employed in this study. Furthermore, similar RC during both sinus rhythm and $\mathrm{AP}$ in 2 cases suggested AP employed in the present study did not influence $\mathrm{S}-\mathrm{A}$ nodal automaticity and antegrade and retrograde conduction time in these cases.

Observation of type I response in 1 case with sinus arrthyhmias (case No. 3), in whom RC was variable and inconsistent during sinus rhythm and became constant during AP, suggested that the shift of pacemaker site in the sinoatrial node was abolished by $\mathrm{AP}$, resulting in one dominant pacemaker site with constant automaticity and conduction time. Disappearance of compensatory zone in these 3 cases during AP suggested continuous suppression of the sinus node by AP. ${ }^{\text {') }}$

Type II response, observed in 2 cases with SSS represented depressive effect of 5 beats of AP with PAS on the sinoatrial node automaticity. Prolonged $S R T_{\max }$ in these 2 cases seems to be in accordance with above explanation. Depressing effect of single supraventricular premature beat on the pacemaker in the sinoatrial node has been demonstrated as a mechanisn causing transient cardiac standstill by Dressler. ${ }^{13)}$

Type III sinoatrial response may be interpreted as a representation of "partial interpolation" and an increase in " the zone of partial interpolation during AP". This phenomenon was observed only with PAS during AP, not during sinus rhythm in the present study. Similar responses have been observed in experimental animals. Drury et al ${ }^{14}$ ) demonstrated progressive shortening of RG in linear fashion as the prematurity of PAS was increased in the dog utilizing extrastimulus technique combined with atrial driving. Eccles et $a .^{15)}$ studying the disturbance of rhythm of the sinoatrial node in the cat produced by 2 successive premature beats, observed the shortening of subsequent cycles and assumed the discharges only a part of the sinoatrial node by an early premature beat. With the simultaneous recording of transmembrane potentials from the sinoatrial node and bipolar right atrial electrogram in the study on the effects of PAS on sinus rhythm in the rabbit, Klein et al ${ }^{11}$ ) 
demonstrated that progressive shortening of sinus return cycle could occur with increasing prematurity of PAS. They suggested that shortening of RC might be resulted from following mechanisms; reduced level of maximum diastolic potential following earlier PAS or decreased duration of premature sinoatrial nodal action potential and/or transient shift of pacemaker within the sinoatrial node. These data in the previous studies in experimental animals may support the assumption that type III sinoatrial response in men may be resulted from shortening of the subsequent sinus cycles after PAS when combined with AP.

An alternative mechanism responsible for the progressive shortening of $\mathrm{RC}$ with increasing prematurity of PAS is based on the assumption that changes in retrograde and antegrade conduction can be induced by PAS during AP. It has been assumed that during partial interpolation PAS following AP does not reset the sinoatrial node, but does prolong the antegrade conduction time of next sinus beat because of concealed conduction of PAS to the perinodal fiber. ${ }^{16)}$ Dhingra et al $^{9}$ ) demonstrated that shortening of cycle length markedly facilitated interpolation and resulted in lengthening the zone of interpolation in man. These assumptions are consistent with the observation by Langendorf et $\mathrm{al}^{17)}$ of one patient with atrial parasystole in whom delay in sinoatrial conduction after interpolation of ectopic atrial beat was demonstrated.

Re-entry of impulses through the sinoatrial node or perinodal area has been shown to produce reciprocal depolarization of the atrium. ${ }^{18)}$ We have observed similar responses after very early PAS in some cases in the present study. These responses are different from what is designated as type III response in the following points: PAS which initiated reentry was delivered very early in diastole and shortening of RC was more marked and precipitous with sinus node reciprocation than it was in type III. In type III response, shortening of RC was gradual with the increase in the prematurity of PAS and "the zone of partial interpolation" accounted for approximately 20 to $50 \%$ of sinus cycle length. $\mathrm{RC}$ in sinus reentry was shorter than SCL, while RC in type III exceeded SCL.

The occurrence of type III resopnse in two-thirds of aged cases included in the present study, regardless of the presence or absence of clinical manifestations of sinus node dysfunction, may suggest the prevalence of disturbed sinoatrial node function and/or sinoatrial conduction in aged patients without full-blown clinical features of sick sinus syndrome. Then the procedure of combined AP and PAS may be of potential clinical value in assessing the sinus node dysfunction in man. However, it has been shown in experimental animals that the atrial activity does not faithfully reflect the events within 
the sinoatrial node. ${ }^{11)}$ In view of the fact that characteristics of sinoatrial nodal action potential, shifts in the pacemaker site within the sinoatrial node and sinoatrial conduction time are important in the determination of altered sinoatrial response to PAS, further study in man will be required to evaluate the usefulness of this type of cardiac stimulation in the study of sin us node dysfunction.

\section{REFERENCES}

1. Mandel WJ, Hayakawa H, Allen HN, Danzig R, Kermaier AI: Assessment of sinus node function in patients with the sick sinus syndrome. Circulation 46: 761, 1972

2. Strauss HC, Saroff AL, Bigger JT, Giardina EGV: Premature atrial stimulation as a key to the understanding of sinoatrial conduction in man. Circulation 47: 86, 1973

3. Steinbeck G, Luderitz B: Comparative study of sinoatrial conduction time and sinus node recovery time. Brit Heart J 37: 956, 1975

4. Lee CH, Cohen LS, Cannom DS: Limitations of sinoatrial recovery time in assessing sinus node dysfunction. Circulation 52 (Suppl): II-12, 1975 (abstract)

5. Reiffel JA, Bigger JT, Konstam MA: The relationship between sinoatrial conduction time and sinus cycle length during spontaneous sinus arrhythmia in adults. Circulation 50: 924, 1974

6. Okimoto T, Ueda K, Kamata C, Yoshida H, Ohkawa S, Hiraoka K, Kuwajima I, Sugiura M, Murakami M, Matsuo $\mathrm{H}$ : Sinus node recovery time and abnormal posipacing phase in the aged patients with sick sinus syndrome. Jap Heart J 17: 290, 1976

7. Ueda K, Kamata C, Matsuo H, Ohkawa S, Okimoto T, Sugiura M: A study on sinoatrial conduction in the aged. Jap Heart J 18: 143, 1977

8. Strauss HC, Bigger JT, Saroff AL, Giardina EGV: Electrophysiologic evaluation of sinus node function in patients with sinus node dysfunction. Circulation 53: 763, 1976

9. Dhingra R, Wyndham C, Amat-Y-Leon F, Denes P, Su D, Rosen KM: Sinus nodal responses to atrial extrastimuli in patients without apparent sinus node disease. Am J Cardiol 36: 445, 1975

10. Denes $P$, Wu D, Dhingra R, Pietras RJ, Rosen KM: The effects of cycle length on cardiac refractory periods in man. Circulation $49: 32,1974$

11. Klein HO, Singer DH, Hoffman BF: Effects of atrial premature systoles on sinus rhythm in the rabbit. Circulat Res 32: 480, 1973

12. Ticzon AR, Strauss HC, Gallagher JJ, Wallace AG: Sinus nodal function in the intact dog heart evaluated by premature atrial stimulation and atrial pacing. Am J Cardiol 35: 492, 1975

13. Dressler W: Prolonged depressing effect of premature supraventricular beats. Am Heart J 72: 25, 1966

14. Drury AN, Brow GR: Observations relating to the unipolar electrical curves of heart muscle with especial reference to the mammalian auricle. Heart 12: 321,1926

15. Eccles JC, Hoff HE: The rhythm of the heart beat. III. Disturbances of rhythm produced by early premature beats. Proc Roy Soc Lond Series B 115: 352, 1934

16. Strauss $\mathbf{H C}$, Bigger JT: Electrophysiological properties of the rabbit sino-atrial perinodal fibers. Circulat Res 31 : 490, 1972

17. Langendorf $R$, Lesser ME, Plotkin P, Levin BD: Atrial parasystole with interpolation. Observations on prolonged sinoatrial conduction. Am Heart J 63: 649, 1962

18. Childers RW, Arnsdorf MF, de la Fuente DJ, Gambetta M, Svenson R: Sinus nodal echoes. Clinical case report and canine studies. Am J Cardiol 31:220, 1973 